\title{
Type D, in Association with Quality of Life in Patients with Multiple Sclerosis
}

\author{
Afsaneh Akhani ${ }^{1}$, Mani Bahrami Monajemi ${ }^{2, ~}{ }^{*}$, Mojtaba Habibi ${ }^{3}$ \\ ${ }^{1}$ Tehran Psychiatric Institute, Iran University of Medical Sciences, Tehran, Iran \\ ${ }^{2}$ MBPSs, Department of Psychology, University of Tehran, Tehran, Iran \\ ${ }^{3}$ Tehran Psychiatric Institute, Iran University of Medical Sciences, Tehran, Iran
}

\section{Email address:}

mani.b.monajemi@warwickgrad.net (M. B. Monajemi), mani.b.monajemi@ut.ac.ir (M. B. Monajemi)

${ }^{*}$ Corresponding author

\section{To cite this article:}

Afsaneh Akhani, Mani Bahrami Monajemi, Mojtaba Habibi. Type D, in Association with Quality of Life in Patients with Multiple Sclerosis. European Journal of Preventive Medicine. Vol. 5, No. 5, 2017, pp. 77-82. doi: 10.11648/j.ejpm.20170505.15

Received: July 14, 2017; Accepted: July 19, 2017; Published: September 28, 2017

\begin{abstract}
Objective: According to recent literatures, Type-D personality, physically and psychologically is tangled with quality of life. Aim of current study is to assess psychological and physical aspects of quality of life among patients with multiple sclerosis. Materials and Methods: In current study, 113 patients were included, who were chosen from MS center (Tehran, Iran). After acquiring written consent form by participants, they were asked to answer to Type D Scale-14 (DS14), Hospital Anxiety and Depression Scale (HADS), Illness Perception Questionnaire (IPQ-SF), Health Status Questionnaire (SF36) and Expanded Disability Status Scale (EDSS). Results: From 113 MS patients, 48(42.5\%) individuals had Type-D personality type. Results were demonstrative of meaningful impact of this group on quality of life variables, depression and anxiety, illness perception and disability status $\left(p<0.001, \eta^{2}=0.29\right)$. Furthermore, results showed that depression, anxiety, illness perceptions and disability status maintained higher mean score among Type-D individuals in comparing to individuals other than Type-D; former group showed lower quality of life in comparing to latter $(\mathrm{p} \leq 0.05)$. Conclusion: With respect to results of current study, MS patients with Type D personality have lower psychological quality of life (physically, psychologically) in comparing to patients with personality other than Type D. Latter group experience higher level of disability in comparing to former group. It appears plausible that notifying personality type of MS patients, can efficiently influence the intervention and clinical implication of these patients.
\end{abstract}

Keywords: Multiple Sclerosis, Type D, Illness Perception, Quality of Life, Disability Status

\section{Introduction}

Multiple sclerosis (MS) is a nervous system disease that tampers with brain and spinal cord function. It harms the myelin sheath, as protectors of your nerve cells. This problem decreases speed of signal transformation between your brain and your body, leading to the symptoms of MS. They can include

(1) Visual problems

(2) Muscle weakness

(3) Difficulty with coordination and balance

(4) Sensations such as numbness, prickling

(5) Thinking and memory problems

To this point, there is no consensus among clinicians and neuroscientists, regarding exact etiology of MS. It may be an autoimmune disease, which happens when mistakenly, immune system attacks healthy cells of the body. Incidence rate of Multiple sclerosis is higher among women. Onset of symptoms is usually between the ages of 20 and 40; in most cases, the disease course is mild and manageable, but some patients may lose their ability to write, communicate or even walk [1].

Diagnosis of MS is not easy and doctor cannot pass comment regarding diagnosis based on the result of one test, diagnosis is made according to medical history, physical exam, neurological exam, MRI, and some other tests (such as Lumbar Puncture). Unfortunately, there is no single gold standard approach with respect to MS treatment, however 
pharmacotherapy may slow down the progression of MS and may alleviate symptoms; physical and occupational therapy may also be helpful [1]. Former studies shows mushrooming trend of this disease in Iran [2]. Sahraian and Colleagues (2010) showed that prevalence of MS is 51.9 among 100.000 people in Iran population [3]. This disease, detrimentally effect sensorimotor, cognitive aspects of human body and subsequently it will lead to physical and psychological wellbeing of these patients [4]. Although, symptoms are not the same in all patients. Various factors influence the quality of life in MS patients; one of them is psychological factors $[5,6]$. According to various literatures, MS patients tend to experience different level of malady, which stems from varied characteristics such as emotions, mental structure cognitive deficiencies and different abilities in handling external circumstances $[7,11]$.

Personality types tend to play a substantial role in terms of characteristics of individuals and their approach toward life events; personality traits may positively/negatively influence our perception of events [7]. Type D personality, a concept used in the field of medical psychology, is defined as the dual predisposition towards negative affectivity (e.g. worry, irritability, gloom) and social inhibition (e.g. reticence and a lack of self-assurance). The letter D stands for "distressed". The Type D personality or the distressed personality is the type of personality characterized by both a negative attitude towards life combined with the person's preference to suppress his emotions.

Type D personalities tend to have negative prospect and pessimistic view toward life events and future incidence.

Due to suppression of emotions, and inhibition from social interactions, they are more prone to experience depression. Type D personalities usually have a low self esteem and a great fear of disapproval and this is the primary reason that prevents them from opening up to others. Therefore, Type D tend to avoid social interaction, not due to lack of interest, because they fear rejection. Negative emotions such as worrying, stress, depression and anger visit type D personalities more often. A small event that is usually overlooked by others can bother a type $\mathrm{D}$ a lot and even ruin his mood $[12,13]$.

Being exposed to of long term negative emotions among Type D individuals will lead to malfunctioning of autonomic nervous system and subsequently this will open gates to more chronic diseases and negative prognostic results $[14,15]$. Other studies have discussed role of Type D in various diseases such as Asthma [16], Fibromyalgia [17], Cancer [18] and Obstruct Sleep Apnea [19]; aforementioned studies has reported poor prognosis and lower quality of life among patients with Type D personality [20, 21]. Notifying Type D detrimental effects on course of the disease, it appears plausible that Type D may play a crucial rule among MS patients as well; and it may lead to lower level of quality of life and disabilities. With respect to social withdrawal among Type D individuals, they tend to suppress their emotions and consequently they experience higher level of stress [22]. Furthermore, Type D tend to have problems regarding managing their emotions; they manage their emotions in avoidant and passive manner which will lead to elevated level of stress [23].

All in all, it appears plausible that Type D patients with MS tend to suffer higher level of anxiety and depression with more disabilities; consequently they will experience lower physical/psychological level of quality of life. Aim of current study to assess aforementioned hypothesis, hopefully results of current study may help clinicians to consider proper psychological interventions, in order to manage and alleviate detrimental synergic effects of Type D personality among MS patients.

\section{Materials and Methods}

This study is casual-comparative study, consisted of MS 113 patients, who were chosen via convenient sampling method from Tehran MS Center. From 113 MS patients, 48 $(42.5 \%)$ individuals had Type-D personality type. Results were demonstrative of meaningful impact of this group on quality of life variables, depression and anxiety, illness perception and disability status $\left(p<0.001, \eta^{2}=0.29\right)$. Furthermore, results showed that depression, anxiety, illness perceptions and disability status maintained higher mean score among Type-D individuals in comparing to individuals other than Type-D; former group showed lower quality of life in comparing to latter $(\mathrm{p} \leq 0.05)$. In this study, groups were paired with respect to age, gender and educational status.

Tools:

a DS-14: Type D personality can be assessed by means of a valid and reliable 14-item questionnaire, the Type D Scale (DS14). Seven items refer to negative affectivity, and seven items refer to social inhibition. People who score 10 points or more on both dimensions are classified as Type D. Bagherian and Bahrami (2011) examined the reliability and validity of the Persian version of 14-item Type D personality scale (DS14). High internal consistency of Cronbach's alpha coefficient has been found to be 0.84 in patients and 0.87 in healthy people for the NA sub-scale and 0.86 in patients and 0.75 in healthy people for the SI sub-scale. The construct validity of NA and SI sub scales were confirmed against neuroticism $(\mathrm{r}=0.65)$ and extroversion $(\mathrm{r}=-0.62)$ subscales of Eysenck questionnaire respectively. Persian version of DS14 is an acceptable, reliable and valid measure of dimensions of Type D personality [24]. In current study, Chronbach's alpha was 0.86 and 0.78 with respect to negative affective and social inhibition respectively.

b HADS: Hospital Anxiety and Depression Scale (HADS) was initially made by Zigmond and Snaith (1983) and is usually used by clinicians to assess the levels of anxiety and depression among patients. The HADS contains of fourteen items; seven of the items relate to anxiety and seven relate to depression. This scale is exceptionally useful in assessing somatic 
symptoms of illness, for example fatigue and insomnia or hypersomnia. Thus, it was hoped, would create a tool for the detection of anxiety and depression in people with physical health problems. According to Bjelland an Colleagues (2002), most factor analyses demonstrated a two-factor solution in good accordance with the HADS subscales for Anxiety (HADS-A) and Depression (HADS-D), respectively. The correlations between the two subscales varied from.40 to.74 (mean.56). Cronbach's alpha for HADS-A varied from.68 to.93 (mean.83) and for HADS-D from.67 to.90 (mean.82). In most studies an optimal balance between sensitivity and specificity was achieved when caseness was defined by a score of 8 or above on both HADS-A and HADS-D. The sensitivity and specificity for both HADS-A and HADS-D of approximately 0.80 were very similar to the sensitivity and specificity achieved by the General Health Questionnaire (GHQ). Correlations between HADS and other commonly used questionnaires were in the range. 49 to. $83[25,26]$. In current study, Chronbach's alpha was 0.80 and 0.83 with respect to depression and anxiety respectively.

c Brief IPQ: The Brief Illness Perception Questionnaire is a 9-item questionnaire was made in order to quickly assess cognitive and emotional representations of illness. The Brief IPQ uses a single-item scale approach to assess perception on a 0-10 response scale. The Brief IPQ comprises 5 items on cognitive representation of illness perception: consequences, timeline, personal control, treatment control, and identity [27, 28, 29]. Former literatures, reported Chronbach's alpha as 0.84 among Iranian population. In current study, we estimated Chronbach's alpha as 0.72 .

d (EDSS): Expanded Disability Status Scale is frequently used in clinical settings regarding assessment disability of patients. EDSS is a method of quantifying disability in multiple sclerosis and monitoring changes in the level of disability over time. It is widely used in clinical trials and in the assessment of people with MS. The scale was developed by a neurologist called John Kurtzke in 1983 as an development from his previous 10 step Disability Status Scale (DSS). The EDSS scale ranges from 0 to 10 in 0.5 unit increments that represent higher levels of disability. Scoring is based on an examination by a neurologist. EDSS steps 1.0 to 4.5 refer to people with MS who are able to walk without any aid and is based on measures of impairment in eight functional systems (FS):

1. Pyramidal - weakness or difficulty moving limbs

2. Cerebellar - ataxia, loss of coordination or tremor
3. Brainstem problems with speech, swallowing and nystagmus

4. sensory - numbness or loss of sensations

5. Bowel and bladder function

6. Visual function

7. Cerebral (or mental) functions

8. Other [30]

e Quality of Life Questionnaire: The Short Form [36] Health Survey is a 36-item, patient-reported survey of patient health. The SF-36 is a measure of health status and an abbreviated variant of it, the SF-6D, is commonly used in health economics as a variable in the quality-adjusted life year calculation to determine the cost-effectiveness of a health treatment. The SF-36 consists of eight scaled scores, which are the weighted sums of the questions in their section. Each scale is directly transformed into a $0-100$ scale on the assumption that each question carries equal weight. The lower the score the more disability. The higher the score the less disability i.e., a score of zero is equivalent to maximum disability and a score of 100 is equivalent to no disability. Former studies postulated that Chronbach's alpha of this questionnaire is between 0.77 to 0.90 among different subscales, however Chronbach's alpha of Vitality is 0.65 [38]. According to Habibi and Colleagues (2013), Chronbach's alpha of subscales was estimated lower than 0.70 [39]. In current study, Chronbach's alpha estimated between 0.69 to 0.91 among all 8 subscales and with respect to physical health and mental health Chronbach's alpha were 0.76 .

\section{Results}

In current study, we chose 113 patients via convenient sample method. Among this sample group, 65 (57/5\%) patients were non-Type D personality and 48 (42.5\%) patients were Type-D personality. Among Type D Group, 39 $(81.2 \%)$ were female and $9(18.8 \%)$ were male; non-Type-D Group, $47(72.2 \%)$ were female and $18(27.7 \%)$ were male. Mean age of Type-D and Non-Type-D group were 30.58 and 30.31 respectively; covariance results showed meaningful effect of age variable on dependent variables $(\mathrm{F}(6$, 103) $=2.29, \mathrm{P}=0.04, \eta^{2}=0.12$ ) Furthermore, mean educational level in both group was diploma. With respect to pairing study groups, chi-square test was used and no meaningful difference was found between two groups with respect to gender $\left(\mathrm{x}^{2}=1.21, \mathrm{df}=1, \mathrm{P}=0.27\right)$ and educational level $\left(\mathrm{x}^{2}=\right.$ 1.21, $\mathrm{df}=1, \mathrm{P}=0.27)$. Furthermore, with respect to paining groups according to age $\mathrm{T}$ test was used and no meaningful difference was noticed $(\mathrm{T}=0.19, \mathrm{df}=111, \mathrm{P}=0.84)$ (Table 1).

Table 1. Satistical Demographic of Type-D and non-Type-D group.

\begin{tabular}{lll}
\hline Variable & Type D & None-type D \\
\hline Gender: frequency (percent) & & \\
Male & $9(18 / 8 \%)$ & $18(27 / 7 \%)$ \\
Female & $39(81 / 2 \%)$ & $47(72 / 2 \%)$ \\
Education, n (\% within group) & & \\
\hline
\end{tabular}




\begin{tabular}{lll}
\hline Variable & Type D & None-type D \\
\hline Middle school & $12(25 \%)$ & $9(13 / 9 \%)$ \\
High school & $16(33 / 3 \%)$ & $24(36 / 9 \%)$ \\
Bachelor & $18(37 / 5 \%)$ & $31(47 / 7 \%)$ \\
Above & $2(4 / 2 \%)$ & $1(1 / 5 \%)$ \\
Age, mean year (SD) & $30 / 58(7 / 04)$ & $30 / 31(7 / 42)$ \\
EDSS, mean (SD) & $1 / 99(0 / 83)$ & $1 / 72(0 / 76)$ \\
Depression, mean (SD) & $9 / 62(4 / 91)$ & $5 / 34(3 / 49)$ \\
Anxiety, mean (SD) & $10 / 42(4 / 69)$ & $5 / 80(3 / 71)$ \\
Illness perception, mean (SD) & $40 / 94(12 / 43)$ & $33 / 52(11 / 69)$ \\
Mental QOL, mean (SD) & $52 / 41(20 / 63)$ & $65 / 78(17 / 54)$ \\
Physical QOL, mean (SD) & $54 / 84(20 / 39)$ & $67 / 87(20 / 23)$ \\
\hline
\end{tabular}

Via MANCOVA we assessed influence of group variable (Type D group, non-Type D group) on subscales (EDSS, Depression, Anxiety, Illness perception, Mental QOL, Physical QOL). $\mathrm{M}$ box results confirms congruity of variance-covariance matrix $(\mathrm{F}(21,37643 / 6)=0 / 89, \mathrm{p}=/ 48)$.
In order assess influence of group variable (Type D group, non-Type D group) and age variable on subscales (EDSS, Depression, Anxiety, Illness perception, Mental QOL, Physical QOL) Anova was used. Details can be noticed in Table 2.

Table 2. ANOVA Result of influence of Variable on different Sub-Scales.

\begin{tabular}{|c|c|c|c|c|c|c|}
\hline Effect & Dependent variable & Mean square & DF & $\mathbf{F}$ & P-value & Effect size \\
\hline \multirow{5}{*}{ Group } & EDSS & $2 / 07$ & $1-108$ & $3 / 64$ & $0 / 05$ & $0 / 03$ \\
\hline & Depression & $476 / 49$ & $1-108$ & $27 / 75$ & $0 / 001$ & $0 / 20$ \\
\hline & Anxiety & $545 / 09$ & $1-108$ & $31 / 94$ & $0 / 001$ & $0 / 22$ \\
\hline & Mental QOL & $4415 / 09$ & $1-108$ & $12 / 38$ & $0 / 001$ & $0 / 103$ \\
\hline & Physical QOL & $4175 / 07$ & $1-108$ & $10 / 83$ & $0 / 001$ & 0/091 \\
\hline \multirow{5}{*}{ Age } & EDSS & $7 / 07$ & $1-108$ & $12 / 46$ & $0 / 001$ & $0 / 10$ \\
\hline & Anxiety & $8 / 43$ & $1-108$ & 494 & $0 / 49$ & $0 / 01$ \\
\hline & Illness perception & $688 / 49$ & $1-108$ & $4 / 98$ & $0 / 03$ & $0 / 04$ \\
\hline & Mental QOL & $707 / 71$ & $1-108$ & $4 / 80$ & $0 / 16$ & $0 / 02$ \\
\hline & Physical QOL & $1850 / 69$ & $1-108$ & $1 / 98$ & $0 / 03$ & $0 / 04$ \\
\hline
\end{tabular}

According to Table 2, there is meaningful difference between two groups (Type D group, non-Type D group) with respect to subscales EDSS, Depression, Anxiety, Illness perception, Mental QOL, Physical QOL. As it can be inferred from mean differences (Table 1), Type D personality maintains higher score with respect to EDSS, Depression, Anxiety, Illness perception and lower scores with respect to
Mental QOL and Physical QOL. Furthermore, age plays a significant role in scores of EDDS, Physical QOL and Illness perception.

Multiple linear Regression analysis was used in order to assess impact of age variable on EDDS, Physical QOL and Illness perception (Table 3 ).

Table 3. Multiple linear Regression analysis of age on EDSS, Illness perception and Physical QOL.

\begin{tabular}{|c|c|c|c|c|c|c|c|c|}
\hline & \multicolumn{4}{|c|}{ Type D } & \multicolumn{4}{|c|}{ None-type D } \\
\hline & $\mathbf{R}^{2}$ & $\beta$ & $\mathbf{T}$ & p-value & $\mathbf{R}^{2}$ & $\beta$ & $\mathbf{T}$ & p-value \\
\hline EDSS & $0 / 043$ & $0 / 21$ & $1 / 44$ & $0 / 15$ & $0 / 19$ & $0 / 43$ & $3 / 83$ & $0 / 001$ \\
\hline Illness perception & $0 / 026$ & $0 / 16$ & $1 / 10$ & $0 / 27$ & $0 / 09$ & $0 / 30$ & $2 / 52$ & $0 / 014$ \\
\hline Physical QOL & $0 / 046$ & $-0 / 21$ & $1 / 48$ & $-0 / 14$ & $0 / 09$ & $-0 / 31$ & $-2 / 55$ & $0 / 013$ \\
\hline
\end{tabular}

As it can be inferred from Table 3, influence of age variable on EDDS, Physical QOL and Illness perception is only meaningful among patients in non-Type-D group, as with aging these patients experience higher level of disability and lower physical QOL. Age variable doesn't have meaningful impact on subscales in Type-D group.

\section{Discussion}

According to results of current study, $42.5 \%$ of MS patients had Type-D personality. This number is demonstrative of high prevalence of Type-D among MS patients. In consistent with the results of current study,
Bagherian and Colleagues postulated that among 191 healthy individuals in their study $24.6 \%$ of them were Type-D personality formation and among participants with a history of Myocardial Infarction (MI), 35.8\% of them were Type-D $[30,31]$. Results of study, also showed that MS patients with Type-D personality, suffer from higher level of disability and lower level of QOL. These results are consistent with the results of the study conducted by Denollet and Conraads (2011); in their study among MI patients, Type-D detrimentally tampered with course of this disease: Firstly, by influencing HPA axis and increasing activity of this axis [8, 32, 33]; secondly, by interfering with nervous system and causing chaos; thirdly, by elevating oxidative stress [9] ratio 
over antioxidants [10]. Hence, it appears plausible that aforementioned triads, plays major role in coarse of MS. With respect to HPA malfunctioning, Kern and Colleagues (2012) postulated that MS patients with higher score in EDDS, demonstrate significant higher level of Cortisol Awakening Response [34]. Oxidative maintains chief role in brain trauma, more specifically among age related brain diseases and MS progression stage. This effect will be intensified as the time goes by and may cause demyelination and neuron regression [35]. Hence it appears plausible, that Type-D personality would indirectly interfere with neuron immune-system functioning, and this disruption would lead to lower score in physical and mental quality of life, which finally will lead to devastating and antagonistic clinical outcome [36, 37].

All in all, it appears plausible, since large proportion of our studies population is consisted of Type-D personality and this type maintains synergic influence with respect to various dimensions of MS patients, hence assessment and tailoring proper intervention approach with respect to personality traits of this group of patients is vital. Impeccable intervention can dramatically influence the clinical outcome and QOL among these group of patients.

\section{Authors' Contributions}

MBM conceived and designed the evaluation. AA and MBM collected and interpreted the clinical data and drafted the manuscript. $\mathrm{MH}$ Participated in conducting statistical evaluation. All authors read and approved the final manuscript.

\section{Acknowledgements}

We would like to thank all patients who participated in the study more specifically all authorities in MS center (mscenter.ir).

\section{References}

[1] Atkins G, Amor S, Fletcher J, Mills K. The biology of multiple sclerosis: Cambridge University Press; 12.

[2] Izadi S, Nikseresht A, Sharifian M, Sahraian MA, Jahromi AH, Aghighi M, et al. Significant increase in the prevalence of multiple sclerosis in iran in 2011. Iranian journal of medical sciences. 2014; 39 (2): 152.

[3] Sahraian MA, Khorramnia S, Ebrahim MM, Moinfar Z, Lotfi J, Pakdaman H. Multiple sclerosis in Iran: a demographic study of 8,000 patients and changes over time. European neurology. 2010; (6): 331-6.

[4] Janssens ACJW, van Doorn PA, de Boer JB, van der Meché FGA, Passchier J, Hintzen RQ. Impact of recently diagnosed multiple sclerosis on quality of life, anxiety, depression and distress of patients and partners. Acta Neurologica Scandinavica. 2003; 108 (6): 389-95.

[5] Warren S, Warren K, Cockerill R. Emotional stress and coping in multiple sclerosis (MS) exacerbations. Journal of psychosomatic research. 1991; 35 (1): 37-47.

[6] Akhani A, Habibi M, Izadikhah Z, Monajemi MB, Jamshinejad N. Quality of Life Model in Multiple Sclerosis: Personality, Mood Disturbance, Catastrophizing and Disease Severity. 2015.

[7] Rätsep T, Kallasmaa T, Pulver A, Gross-Paju K. Personality as a predictor of coping efforts in patients with multiple sclerosis. Multiple Sclerosis. 2000; 6 (6): 397-402.

[8] Benedict RH, Zivadinov R. Risk factors for and management of cognitive dysfunction in multiple sclerosis. Nature Reviews Neurology. 2011; 7 (6): 332-42.

[9] Goretti B, Portaccio E, Zipoli V, Razzolini L, Amato MP. Coping strategies, cognitive impairment, psychological variables and their relationship with quality of life in multiple sclerosis. Neurological Sciences. 2010; 31: 227-30.

[10] Akhani A, Izadikhah Z, Bagherian-Sararoudi R, Khorvash F. Investigating the effect of illness perception on the relationship between early maladaptive schemas and level of motor disability in multiple sclerosis patients. J Res Behav Sci. $2013 ; 10$ (7): 609-18.

[11] Goretti B, Portaccio E, Zipoli V, Hakiki B, Siracusa G, Sorbi $\mathrm{S}$, et al. Coping strategies, psychological variables and their relationship with quality of life in multiple sclerosis. Neurological Sciences. 2009; 30 (1): 15-20.

[12] Dornelas EA. Stress Proof the heart: Springer; 2012.

[13] Kupper N, Kessing D, Denollet J. Recent progress in understanding the clinical concept of Type D. Journal of Psychosomatic Research. 2013; 2 (75): 197-8.

[14] Denollet J, Conraads VM. Type D personality and vulnerability to adverse outcomes in heart disease. Cleveland Clinic journal of medicine. 2011; 78 Suppl 1: S13.9-.

[15] Denollet J, Conraads VM, Brutsaert DL, De Clerck LS, Stevens WJ, Vrints CJ. Cytokines and immune activation in systolic heart failure: the role of Type D personality. Brain, Behavior, and Immunity. 2003; 17 (4): 304-9.

[16] Kim SR, Kim HK, Kang JH, Jeong SH, Kim HY, Kim SR, et al. Does type D personality affect symptom control and quality of life in asthma patients? Journal of clinical nursing. 2015; 24 (5-6): 739-48.

[17] Van Middendorp H, Kool MB, van Beugen S, Denollet J, Lumley MA, Geenen R. Prevalence and relevance of Type D personality in fibromyalgia. General Hospital Psychiatry. 2015.

[18] Mols F, Thong MS, van de Poll-Franse LV, Roukema JA, Denollet J. Type D (distressed) personality is associated with poor quality of life and mental health among 3080 cancer survivors. Journal of affective disorders. 2012; 136 (1): 26-34.

[19] Broström A, Strömberg A, Mårtensson J, Ulander M, Harder L, Svanborg E. Association of Type D personality to perceived side effects and adherence in CPAP - treated patients with OSAS. Journal of sleep research. 2007; 16 (4): 439-47.

[20] Van Dooren FEP, Verhey FRJ, Pouwer F, Schalkwijk CG, Sep SJS, Stehouwer CDA, et al. Association of Type D personality with increased vulnerability to depression: Is there a role for inflammation or endothelial dysfunction? - The Maastricht Study. Journal of Affective Disorders. 2016; 189: 118-25. 
[21] Williams L, O'Connor RC, Grubb NR, O'Carroll RE. Type D personality and illness perceptions in myocardial infarction patients. J Psychosom Res. 2011; 70 (2): 141-4.

[22] Bibbey A, Carroll D, Ginty AT, Phillips AC. Cardiovascular and Cortisol Reactions to Acute Psychological Stress Under Conditions of High Versus Low Social Evaluative Threat: Associations With the Type D Personality Construct. Psychosomatic Medicine. 2015; 77 (5): 599-608.

[23] Polman R, Borkoles E, Nicholls AR. Type D personality, stress, and symptoms of burnout: The influence of avoidance coping and social support. British Journal of Health Psychology. 2010; 15 (3): 681-96.

[24] Reza Bagherian, PhD•, 1 and Hadi Bahrami Ehsan, PhD2. (2011). Psychometric Properties of the Persian Version of Type D Personality Scale (DS14). Available: https://www.ncbi.nlm.nih.gov/pmc/articles/PMC3939967/. Last accessed Jan 2017.

[25] Zigmond, AS; Snaith, RP (1983). "The hospital anxiety and depression scale". Acta Psychiatrica Scandinavica. 67 (6): 361-370. doi: 10.1111/j.1600-0447.1983.tb09716.x. PMID 6880820 .

[26] Bjelland, I; et al. (2002). "The validity of the Hospital Anxiety and Depression Scale. An updated literature review". Journal of Psychosomatic Research. 52 (2): 69-77. doi: 10.1016/s0022-3999 (01) 00296-3. PMID 11832252.

[27] Broadbent, E. et al. J Psychosom Res. 2006; 60: 631-637.

[28] Chong, J. et al. Dev Med Child Neurol. 2010; 52: 21-22.

[29] Giri, P. et al. Occup Med (Lond). 2009; 59: 550-555.

[30] Cohen RA, Kessler HR, Fischer M. (1993). The Extended Disability Status Scale (EDSS) as a predictor of impairments of functional activities of daily living in multiple sclerosis. Available: https://www.ncbi.nlm.nih.gov/pubmed/8482974. Last accessed Feb 2016. PMID: 8482974
[31] Bagherian R, Bahrami Ehsan H. Psychometric properties of the persian version of Type D Personality Scale (DS14). Iranian journal of psychiatry and behavioral sciences. 2011; 5 (2): $12-7$.

[32] Melief J, de Wit SJ, van Eden CG, Teunissen C, Hamann J, Uitdehaag BM, et al. HPA axis activity in multiple sclerosis correlates with disease severity, lesion type and gene expression in normal-appearing white matter. Acta neuropathologica. 2013; 126 (2): 237-49.

[33] Kümpfel T, Schwan M, Weber F, Holsboer F, Trenkwalder C, Then Bergh F. Hypothalamo-pituitary-adrenal axis activity evolves differentially in untreated versus treated multiple sclerosis. Psychoneuroendocrinology. 2014; 45: 87-95.

[34] Kern S, Krause I, Horntrich A, Thomas K, Aderhold J, Ziemssen T. Cortisol awakening response is linked to disease course and progression in multiple sclerosis: Public Library of Science; 2013.

[35] Lassmann H, van Horssen J. Oxidative stress and its impact on neurons and glia in multiple sclerosis lesions .Biochimica et Biophysica Acta (BBA)-Molecular Basis of Disease. 2015.

[36] Gilgun-Sherki Y, Melamed E, Offen D. The role of oxidative stress in the pathogenesis of multiple sclerosis: the need for effective antioxidant therapy. Journal of neurology. 2004251 (3): 261-8.

[37] Williams L, Abbott C, Kerr R. Health behaviour mediates the relationship between Type D personality and subjective health in the general population. Journal of health psychology. 2015: 1359105315571977.

[38] Montazeri A, Goshtasebi A, Vahdaninia M, Gandek B. The Short Form Health Survey (SF-36): Translation and validation study of the Iranian version. Qual Life Res. 2005; 14(3): 875-82.

[39] Habibi M, Khodaei E, Moghadamzadeh A, Shamsedini S, Barekatain M. Psychometric properties and hierarchical factor structure of short form health survey scale (SF-36) in a nonclinical sample J Res Behav Sci. 2013; 10(6): 472-90. 